\title{
The Existence of a Nontrivial Solution for a $p$-Kirchhoff Type Elliptic Equation in $\mathbb{R}^{N}$
}

\author{
Zonghu Xiu ${ }^{1,2}$ \\ ${ }^{1}$ Science and Information College, Qingdao Agricultural University, Qingdao 266109, China \\ ${ }^{2}$ College of Science, Hohai University, Nanjing 210098, China
}

Correspondence should be addressed to Zonghu Xiu; qingda@163.com

Received 11 March 2013; Revised 18 July 2013; Accepted 18 July 2013

Academic Editor: Dumitru Motreanu

Copyright (C) 2013 Zonghu Xiu. This is an open access article distributed under the Creative Commons Attribution License, which permits unrestricted use, distribution, and reproduction in any medium, provided the original work is properly cited.

Using Mountain Pass lemma, under some appropriate assumptions, we establish the existence of one nontrivial solution for a class of $p$-Kirchhoff-type elliptic equations in $\mathbb{R}^{N}$.

\section{Introduction and Main Result}

In this paper, we consider the existence of solution for the following elliptic problem:

$$
\begin{gathered}
-\left(a+b \int_{\mathbb{R}^{N}} g(x)|\nabla u|^{p} d x\right) \operatorname{div}\left(g(x)|\nabla u|^{p-2} \nabla u\right) \\
+h(x)|u|^{p-2} u=f(x, u), \quad x \in \mathbb{R}^{N}, \\
u(x) \longrightarrow 0 \quad \text { as }|x| \longrightarrow \infty,
\end{gathered}
$$

where $a>1, b>0, N \geq 3, g(x)$, and $h(x)$ are positive functions, $f(x, t): \mathbb{R}^{N} \times R \rightarrow R$ is continuous function, and further assumptions will be listed later. Problems like (1) originally came from the stationary problem of a model introduced by Kirchhoff [1]. Due to the existence of the integration over the whole space, problems like (1) are also called nonlocal problems.

In recent years, the Kirchhoff-type equations with $p$ Laplacian operator has been considered by many authors; see [2-6]. When $h(x) \equiv 0$, the authors in [6] considered the following similar Kirchhoff-type elliptic problem on the bounded domain $\Omega \subset \mathbb{R}^{N}$

$$
\begin{gathered}
-\left[M\left(\int_{\Omega}|\nabla u|^{p} d x\right)\right]^{p-1} \operatorname{div}\left(|\nabla u|^{p-2} \nabla u\right) \\
=f(x, u), \quad x \in \Omega, \\
u(x)=0 \quad \text { on } \partial \Omega .
\end{gathered}
$$

The function $M(t)$ in (2) is required to meet the condition of $m_{0} \leq M(t) \leq m_{1}$ for some constants $m_{0}, m_{1}>0$. The authors proved that problem (2) has at least one positive solution under some other additional conditions. Problem (2) was also considered in [3], where the function $f(x, u)$ is odd about $u$. For $p=2$, we refer to [7-9]. The authors in [8] studied the following Kirchhoff type problem:

$$
\begin{gathered}
-\left(\int_{\Omega} a+b|\nabla u|^{2} d x\right) \Delta u+u=f(x, u), \quad x \in \mathbb{R}^{N}, \\
u(x) \longrightarrow 0 \quad \text { as }|x| \longrightarrow+\infty .
\end{gathered}
$$

By the Fountain theorem, the author proved the existence of infinitely many solutions. Note that one of the assumptions made on the function $f(x, u)$ in (3) is that $f(x, u)=$ $-f(x,-u)$ for any $(x, u) \in\left(\mathbb{R}^{N}, \mathbb{R}\right)$. In the present paper, however, the function $f(x, u)$ is not required to be odd about $u$ as that of $[3,8]$. When $a=b=0$ and $g(x) \equiv$ 1 , problem (1) becomes the $p$-Laplacian elliptic equations without nonlocal term, and this kind of problem is also studied by many authors. For these works, we refer to [10-14] and the references therein. In [15], Liu discussed the following elliptic problem:

$$
\begin{gathered}
-\operatorname{div}\left(|\nabla u|^{p-2} \nabla u\right)+V(x)|u|^{p-2} u=f(x, u), \\
u(x) \in W^{1, p}\left(\mathbb{R}^{N}\right) .
\end{gathered}
$$


The author proved that problem (4) has at least one ground state. The weight function $V(x)$ is required to be bounded; more precisely, there exist constants $\alpha, \beta>0$ such that

$$
V(x) \in C\left(\mathbb{R}^{N}\right), \quad 0<\alpha \leq V(x) \leq \beta<+\infty .
$$

We point out that the weight function $h(x)$ in problem (1) is permitted to tends to infinity.

In this paper, inspired by $[4,8,15]$, we consider the existence of solution of problem (1). By the variational method, we will prove that problem (1) has at least one nontrivial weak solution. Since problem (1) is considered in the whole space $\mathbb{R}^{N}$, the loss of compactness of the Sobolev embedding renders the variational technique more delicate.

In this paper, we make the following hypothesis:

$$
\begin{aligned}
& \left(A_{1}\right) g(x) \geq 1, h(x) \geq h_{0}>0 \text { and } h(x) \rightarrow+\infty \text { as } \\
& |x| \rightarrow \infty,
\end{aligned}
$$

$\left(A_{2}\right)$ there exists $\mu \geq 2 p$ such that $0 \leq \mu F(x, t) \leq$ $t f(x, t)$, where $F(x, t)=\int_{0}^{t} f(x, s) d s$,

$\left(A_{3}\right)$ there exist constants $c>0$ and $p<q<p^{*}$ such that $|f(x, t)| \leq c|t|^{q-1}$.

Remark 1. Throughout this paper, we denote by $c$ the constant which may vary from line to line but remains independent of the relevant quantities. Note that there exist many functions $g(x), h(x)$, and $f(x, t)$ such that satisfy the assumptions of Theorem 3, for example, $g(x)=1+x^{4}, h(x)=e^{x^{2}}, f(x, t)=$ $t^{5 p-1} /\left(1+x^{4}\right)$. of

Let $W_{0}^{1, p}\left(\mathbb{R}^{N}\right)$ be the usual Sobolev space with the norm

$$
\|u\|=\left(\int_{\mathbb{R}^{N}}|\nabla u|^{p}+|u|^{p} d x\right)^{1 / p}
$$

Denote

$$
E=\left\{u \in W_{0}^{1, p}\left(\mathbb{R}^{N}\right): \int_{\mathbb{R}^{N}}\left(|\nabla u|^{p}+h(x)|u|^{p}\right) d x<\infty\right\} .
$$

Then, $E$ is a Sobolev space with the norm of

$$
\|u\|_{E}=\left(\int_{\mathbb{R}^{N}}\left(|\nabla u|^{p}+h(x)|u|^{p}\right) d x\right)^{1 / p} .
$$

We give another space

$$
X=\left\{u \in E: \int_{\mathbb{R}^{N}}\left(g(x)|\nabla u|^{p}+h(x)|u|^{p}\right) d x<\infty\right\}
$$

endowed with the norm

$$
\|u\|_{X}=\left(\int_{\mathbb{R}^{N}}\left(g(x)|\nabla u|^{p}+h(x)|u|^{p}\right) d x\right)^{1 / p} .
$$

It is not difficult to check that $X$ is a Banach space. The Euler functional of problem (1) is

$$
\begin{aligned}
J(u)= & \frac{a}{p} \int_{\mathbb{R}^{N}} g(x)|\nabla u|^{p} d x+\frac{b}{2 p}\left(\int_{\mathbb{R}^{N}} g(x)|\nabla u|^{p} d x\right)^{2} \\
& +\frac{1}{p} \int_{\mathbb{R}^{N}} h(x)|u|^{p} d x-\int_{\mathbb{R}^{N}} F(x) d x,
\end{aligned}
$$

where $F(u)=\int_{0}^{u} f(x, t) d t$. Then, the assumptions $\left(A_{1}\right)-\left(A_{3}\right)$ imply that $J(u) \in C^{1}\left(X, \mathbb{R}^{1}\right)$, and for any $\varphi \in X$, there holds

$$
\begin{aligned}
\left\langle J^{\prime}(u), \varphi\right\rangle= & a \int_{\mathbb{R}^{N}} g(x)|\nabla u|^{p-2} \nabla u \nabla \varphi d x \\
& +b \int_{\mathbb{R}^{N}} g(x)|\nabla u|^{p} d x \int_{\mathbb{R}^{N}} g(x)|\nabla u|^{p-2} \nabla u \nabla \varphi d x \\
& +\int_{\mathbb{R}^{N}} h(x)|u|^{p-2} u \varphi d x-\int_{\mathbb{R}^{N}} f(x, u) \varphi d x .
\end{aligned}
$$

Particularly,

$$
\begin{aligned}
\left\langle J^{\prime}(u), u\right\rangle= & a \int_{\mathbb{R}^{N}} g(x)|\nabla u|^{p} d x \\
& +b\left(\int_{\mathbb{R}^{N}} g(x)|\nabla u|^{p} d x\right)^{2} \\
& +\int_{\mathbb{R}^{N}} h(x)|u|^{p} d x-\int_{\mathbb{R}^{N}} f(x, u) u d x .
\end{aligned}
$$

Definition 2. A function $u \in X$ is said to be a weak solution of (1) if and only if (12) holds for any $\varphi \in X$.

Our main result is listed later.

Theorem 3. Assume $\left(A_{1}\right)-\left(A_{3}\right)$. Then, problem (1) has at least one nontrivial weak solution in $X$.

This paper is organized as follows. In Section 2, we introduce some definitions and prove several lemmas which will be used later. In Section 3, we give the proof of Theorem 3 by making use of the Mountain Pass lemma.

\section{Preliminary Results}

In this section, we give some important lemmas, which will be needed in the proof of our main result. Particularly, one result of compact embedding on unbounded domain will be proved.

Lemma 4. Assume $\left(A_{1}\right)$ and $p \leq q<p^{*}$. Then, the embedding $X \hookrightarrow L^{q}\left(\mathbb{R}^{N}\right)$ is compact.

Proof. We split the proof into two cases.

Case $1(q=p)$. Let

$$
B_{R}=\left\{u \in X:\|u\|_{X}<R\right\}, \quad B_{R}^{c}=\mathbb{R}^{N} \backslash B_{R}
$$


and $\eta=\inf _{B_{R}^{c}} h(x)$; then $\left(A_{1}\right)$ shows that $\eta \rightarrow+\infty$ as $R \rightarrow$ $\infty$. Furthermore, one gets that

$$
\int_{B_{R}^{c}}|u|^{p} d x \leq \eta^{-1} \int_{B_{R}^{c}} h(x)|u|^{p} d x,
$$

which implies that

$$
\begin{aligned}
\left(\int_{B_{R}^{c}}|u|^{p} d x\right)^{1 / p} & \leq \eta^{-1 / p}\left(\int_{B_{R}^{c}} h(x)|u|^{p} d x\right)^{1 / p} \\
& \leq \eta^{-1 / p}\left(\int_{\mathbb{R}^{N}} h(x)|u|^{p} d x\right)^{1 / p} \\
& \leq \eta^{-1 / p}\|u\|_{x} .
\end{aligned}
$$

It follows from (16) that

$$
\lim _{R \rightarrow \infty} \sup _{u \in X \backslash\{0\}} \frac{\|u\|_{L^{p}\left(B_{R}^{c}\right)}}{\|u\|_{X}}=0
$$

Let $\left\{u_{n}\right\}$ be a bounded sequence of $X$ such that $\left\|u_{n}\right\|_{X} \leq c$. Then, there exists $u \in X$ such that $u_{n} \rightarrow u$. For any $\varepsilon>0$, it follows from (17) that there exists $R_{\varepsilon}$ large enough such that

$$
\left\|u_{n}\right\|_{L^{p}\left(B_{R_{\varepsilon}}^{c}\right)} \leq \varepsilon c^{-1}\left\|u_{n}\right\|_{X} \leq \varepsilon \quad(n=1,2, \ldots) .
$$

Note that the embedding $X \hookrightarrow L^{p}\left(B_{R_{\varepsilon}}\right)$ is compact. Therefore, for these $\varepsilon$ and $R_{\varepsilon}$, there exists $N_{\varepsilon}>0$ large enough such that

$$
\left\|u_{n}-u\right\|_{L^{p}\left(B_{R_{\varepsilon}}\right)} \leq \varepsilon
$$

for all $n>N_{\varepsilon}$. Thus, one can get from (18) and (19) that

$$
\begin{aligned}
\left\|u_{n}-u\right\|_{L^{p}\left(\mathbb{R}^{N}\right)} \leq & \left\|u_{n}-u\right\|_{L^{p}\left(B_{R_{\varepsilon}}\right)} \\
& +\left\|u_{n}\right\|_{L^{p}\left(B_{R_{\varepsilon}}^{c}\right)}+\|u\|_{L^{p}\left(B_{R_{\varepsilon}}^{c}\right)}<3 \varepsilon,
\end{aligned}
$$

which implies that $u_{n} \rightarrow u$ strongly in $L^{q}\left(\mathbb{R}^{N}\right)$.

Case $2\left(p<q<p^{*}\right)$. By the Hölder and Young inequalities, one can get from $\left(A_{1}\right)$ that

$$
\begin{aligned}
\int_{B_{R}^{c}}|u|^{q} d x \leq & \left(\int_{B_{R}^{c}}|u|^{p} d x\right)^{\left(p^{*}-q\right) /\left(p^{*}-p\right)} \\
& \times\left(\int_{B_{R}^{c}}|u|^{p^{*}} d x\right)^{(q-p) /\left(p^{*}-p\right)} \\
\leq & \eta^{-\left(p^{*}-q\right) /\left(p^{*}-p\right)}\left(\int_{B_{R}^{c}} h(x)|u|^{p} d x\right)^{\left(p^{*}-q\right) /\left(p^{*}-p\right)} \\
& \times\left(\int_{B_{R}^{c}}|u|^{p^{*}} d x\right)^{(q-p) /\left(p^{*}-p\right)}
\end{aligned}
$$

$$
\begin{aligned}
& \leq c \eta^{-\left(p^{*}-q\right) /\left(p^{*}-p\right)}\left[\left(\int_{B_{R}^{c}} h(x)|u|^{p} d x\right)^{q / p}\right. \\
& \left.+\left(\int_{B_{R}^{c}}|u|^{p^{*}} d x\right)^{q / p^{*}}\right] \\
& \leq c \eta^{-\left(p^{*}-q\right) /\left(p^{*}-p\right)}\left[\left(\int_{B_{R}^{c}} h(x)|u|^{p} d x\right)^{q / p}\right. \\
& \left.+\left(\int_{B_{R}^{c}} g(x)|\nabla u|^{p} d x\right)^{q / p}\right] \\
& \leq c \eta^{-\left(p^{*}-q\right) /\left(p^{*}-p\right)}\|u\|_{X}^{q} .
\end{aligned}
$$

Since $p<q<p^{*}$ and $\eta \rightarrow+\infty$ as $R \rightarrow \infty$, then

$$
\lim _{R \rightarrow \infty} \sup _{u \in X \backslash\{0\}} \frac{\|u\|_{L^{q}\left(B_{R}^{c}\right)}}{\|u\|_{X}}=0 .
$$

Therefore, similar to the proof of Case 1, the embedding $E \hookrightarrow L^{q}\left(\mathbb{R}^{N}\right)$ is compact.

Now, we give the definitions of Palais-Smale (simply $(\mathrm{PS})_{c}$ ) sequence and $(\mathrm{PS})_{c}$ condition.

Definition 5. Let $c \in R^{1}, J(u) \in C^{1}(X, R)$, and $X$ be a Banach space. The sequence $\left\{u_{n}\right\} \subset X$ is said to be a $(P S)_{c}$ sequence if there holds

$$
J\left(u_{n}\right) \longrightarrow c, \quad J^{\prime}\left(u_{n}\right) \longrightarrow 0 \quad \text { in } X^{*} \text { as } n \longrightarrow \infty .
$$

A functional $J(u)$ is said to satisfy the (PS) $)_{c}$ condition if any $(\mathrm{PS})_{c}$ sequence in $X$ contains a convergent subsequence.

Next, we will prove that the functional $J(u)$ satisfies the $(\mathrm{PS})_{c}$ condition.

Lemma 6. Assume $\left(A_{1}\right)-\left(A_{3}\right)$. Then, $J(u)$ satisfies the $(P S)_{c}$ condition on $X$ for any $c \in \mathbb{R}^{1}$.

Proof. Let $\left\{u_{n}\right\}$ be an any (PS) sequence in $X$. We divide the proof into two steps.

Step $1\left(\left\{u_{n}\right\}\right.$ is bounded in $\left.X\right)$. Note that $a>1$; then, it follows from $(23)$ and $\left(A_{2}\right)$ that

$$
\begin{aligned}
c+\left\|u_{n}\right\|_{X} \geq & J\left(u_{n}\right)-\frac{1}{2 p}\left\langle J^{\prime}\left(u_{n}\right), u_{n}\right\rangle \\
= & \left(\frac{1}{p}-\frac{1}{2 p}\right) a \int_{\mathbb{R}^{N}} g(x)\left|\nabla u_{n}\right|^{p} d x \\
& +\left(\frac{1}{p}-\frac{1}{2 p}\right) \int_{\mathbb{R}^{N}} h(x)\left|u_{n}\right|^{p} d x \\
& +\int_{\mathbb{R}^{N}}\left(\frac{1}{2 p} f\left(x, u_{n}\right) u_{n}-F\right) d x
\end{aligned}
$$




$$
\begin{aligned}
\geq & \frac{1}{2 p} \int_{\mathbb{R}^{N}} g(x)\left|\nabla u_{n}\right|^{p} d x \\
& +\frac{1}{2 p} \int_{\mathbb{R}^{N}} h(x)\left|u_{n}\right|^{p} d x=\frac{1}{2 p}\left\|u_{n}\right\|_{X}^{p},
\end{aligned}
$$

which implies that $\left\{u_{n}\right\}$ is bounded in $X$.

Step $2\left(\left\{u_{n}\right\}\right.$ converges strongly in $\left.X\right)$. Since $\left\{u_{n}\right\}$ is bounded in the separable $X$, there exist $u_{0} \in X$ and a subsequence of $\left\{u_{n}\right\}$, still denoted by $\left\{u_{n}\right\}$, such that $u_{n} \rightarrow u_{0}$ in $X$. Now, we want to prove that

$$
\begin{aligned}
& \int_{\mathbb{R}^{N}} f\left(x, u_{n}\right) u_{n} d x \\
& \quad-\int_{\mathbb{R}^{N}} f\left(x, u_{n}\right) u_{0} d x \longrightarrow 0, \text { as } n \longrightarrow \infty .
\end{aligned}
$$

In fact, it follows from $\left(A_{3}\right)$ that

$$
\begin{aligned}
& \left|\int_{\mathbb{R}^{N}} f\left(x, u_{n}\right) u_{n} d x-\int_{\mathbb{R}^{N}} f\left(x, u_{n}\right) u_{0} d x\right| \\
& \quad \leq \int_{\mathbb{R}^{N}}\left|u_{n}\right|^{q-1}\left|u_{n}-u_{0}\right| d x
\end{aligned}
$$

Thus, in order to prove (25), we need only to prove that

$$
\int_{\mathbb{R}^{N}}\left|u_{n}\right|^{q-1}\left|u_{n}-u_{0}\right| d x \longrightarrow 0, \quad \text { as } n \longrightarrow \infty
$$

Here, we divide the proof (27) into two cases:

(i)

$$
p \leq q<\frac{(N-1) p}{N-p}
$$

By the Hölder inequality, we obtain that

$$
\begin{gathered}
\int_{\mathbb{R}^{N}}\left|u_{n}\right|^{q-1}\left|u_{n}-u_{0}\right| d x \\
\leq\left(\int_{\mathbb{R}^{N}}\left|u_{n}\right|^{p(q-1) /(p-1)} d x\right)^{(p-1) / p} \\
\quad \times\left(\int_{\mathbb{R}^{N}}\left|u_{n}-u_{0}\right|^{p} d x\right)^{1 / p}
\end{gathered}
$$

It is easy to check that $p \leq p(q-1) /(p-1)<p^{*}$. Then, the compact embedding in Lemma 4 shows that

$$
\begin{gathered}
\left(\int_{\mathbb{R}^{N}}\left|u_{n}\right|^{p(q-1) /(p-1)} d x\right)^{(p-1) / p}<\infty, \\
\left(\int_{\mathbb{R}^{N}}\left|u_{n}-u_{0}\right|^{p} d x\right)^{1 / p} \longrightarrow 0 \text { as } n \longrightarrow \infty,
\end{gathered}
$$

which gives (27).

(ii)

$$
\frac{(N-2) p}{N-p} \leq q<p^{*} .
$$

Let $\lambda_{1}=p^{*} /(q-2)$. Since $1<p<N$ and $p(N-2) /(N-$ $p) \leq q<p^{*}$, there exist $p \leq \lambda_{2}=\lambda_{3}<p^{*}$ such that

$$
\frac{1}{\lambda_{1}}+\frac{1}{\lambda_{2}}+\frac{1}{\lambda_{3}}=1 .
$$

The Hölder inequality shows that

$$
\begin{aligned}
\int_{\mathbb{R}^{N}}\left|u_{n}\right|^{q-1}\left|u_{n}-u_{0}\right| d x \leq & \left(\int_{\mathbb{R}^{N}}\left|u_{n}\right|^{(q-2) \lambda_{1}} d x\right)^{1 / \lambda_{1}} \\
& \times\left(\int_{\mathbb{R}^{N}}\left|u_{n}\right|^{\lambda_{2}}\right)^{1 / \lambda_{2}} \\
& \times\left(\int_{\mathbb{R}^{N}}\left|u_{n}-u_{0}\right|^{\lambda_{3}} d x\right)^{1 / \lambda_{3}} .
\end{aligned}
$$

Therefore, the embedding in Lemma 4 implies that

$$
\begin{gathered}
\left(\int_{\mathbb{R}^{N}}\left|u_{n}\right|^{(q-2) \lambda_{1}} d x\right)^{1 / \lambda_{1}}<\infty, \\
\left(\int_{\mathbb{R}^{N}}\left|u_{n}\right|^{\lambda_{2}}\right)^{1 / \lambda_{2}}<\infty, \\
\left(\int_{\mathbb{R}^{N}}\left|u_{n}-u_{0}\right|^{\lambda_{3}} d x\right)^{1 / \lambda_{3}} \longrightarrow 0,
\end{gathered}
$$

which also gives (27).

Note that $(N-1) p /(N-p)<p^{*}$ and

$$
\begin{gathered}
\frac{(N-2) p}{N-p} \geq p, \quad p \geq 2, \\
\frac{(N-2) p}{N-p}<p, \quad 1<p<2 .
\end{gathered}
$$

Therefore, the previous cases (i) and (ii) imply that (25) holds for all $p \leq q<p^{*}$.

Denote

$$
\begin{aligned}
D_{n}= & \left\langle J^{\prime}\left(u_{n}\right), u_{n}\right\rangle-\left\langle J^{\prime}\left(u_{n}\right), u_{0}\right\rangle \\
& +\int_{\mathbb{R}^{N}} f\left(x, u_{n}\right) u_{n} d x-\int_{\mathbb{R}^{N}} f\left(x, u_{n}\right) u_{0} d x .
\end{aligned}
$$

Then, it follows from (23) and (25) that $D_{n}=o(1)$ and

$$
\begin{aligned}
o(1)=D_{n}= & a \int_{\mathbb{R}^{N}} g(x)\left|\nabla u_{n}\right|^{p-2} \nabla u_{n} \nabla\left(u_{n}-u_{0}\right) d x \\
& +b\left(\int_{\mathbb{R}^{N}} g(x)\left|\nabla u_{n}\right|^{p} d x\right) \\
& \times \int_{\mathbb{R}^{N}} g(x)\left|\nabla u_{n}\right|^{p-2} \nabla u \nabla\left(u_{n}-u_{0}\right) d x \\
& +\int_{\mathbb{R}^{N}} h(x)\left|u_{n}\right|^{p-2} u_{n}\left(u_{n}-u_{0}\right) d x \\
& +\int_{\mathbb{R}^{N}} f\left(x, u_{n}\right) u_{n} d x-\int_{\mathbb{R}^{N}} f\left(x, u_{n}\right) u_{0} d x
\end{aligned}
$$


Since $u_{n} \rightarrow u_{0}$ in $X$, one obtains from the Hölder inequality that

$$
\begin{aligned}
o(1)= & -a \int_{\mathbb{R}^{N}} g(x)\left|\nabla u_{0}\right|^{p-2} \nabla u_{0} \nabla\left(u_{n}-u_{0}\right) d x \\
& -b\left(\int_{\mathbb{R}^{N}} g(x)\left|\nabla u_{n}\right|^{p} d x\right) \\
& \times \int_{\mathbb{R}^{N}} g(x)\left|\nabla u_{0}\right|^{p-2} \nabla u_{0} \nabla\left(u_{n}-u_{0}\right) d x \\
& -\int_{\mathbb{R}^{N}} h(x)\left|u_{0}\right|^{p-2} u_{0}\left(u_{n}-u_{0}\right) d x .
\end{aligned}
$$

Thus, it follows from (37) and (38) that

$$
\begin{aligned}
o(1)= & a \int_{\mathbb{R}^{N}} g(x)\left(\left|\nabla u_{n}\right|^{p-2} \nabla u_{n}\right. \\
& \left.-\left|\nabla u_{0}\right|^{p-2} \nabla u_{0}\right) \nabla\left(u_{n}-u_{0}\right) d x \\
& +b\left(\int_{\mathbb{R}^{N}} g(x)\left|\nabla u_{n}\right|^{p} d x\right) \\
& \times \int_{\mathbb{R}^{N}} g(x)\left(\left|\nabla u_{n}\right|^{p-2} \nabla u_{n}\right. \\
& +\int_{\mathbb{R}^{N}} h(x)\left(\left|u_{n}\right|^{p-2} u_{n}-\left|u_{0}\right|^{p-2} u_{0}\right)\left(u_{n}-u_{0}\right) d x \\
& +\int_{\mathbb{R}^{N}} f\left(x, u_{n}\right) u_{n} d x-\int_{\mathbb{R}^{N}} f\left(x, u_{n}\right) u_{0} d x .
\end{aligned}
$$

Since $a \geq 1, b>0$, and $g(x), h(x)$ are positive functions, we get from (25) and (39) that

$$
\begin{gathered}
\int_{\mathbb{R}^{N}} g(x)\left(\left|\nabla u_{n}\right|^{p-2} \nabla u_{n}\right. \\
\left.-\left|\nabla u_{0}\right|^{p-2} \nabla u_{0}\right) \nabla\left(u_{n}-u_{0}\right) d x \rightarrow 0, \\
\int_{\mathbb{R}^{N}} h(x)\left(\left|u_{n}\right|^{p-2} u_{n}-\left|u_{0}\right|^{p-2} u_{0}\right)\left(u_{n}-u_{0}\right) d x \rightarrow 0 .
\end{gathered}
$$

Therefore, we can deduce from (40) and the following standard inequalities in [16]:

$$
|\xi-\zeta|^{p} \leq\left\{\begin{array}{cl}
c\left\langle|\xi|^{p-2} \xi-|\zeta|^{p-2} \zeta, \xi-\zeta\right\rangle & \text { for } p \geq 2, \\
c\left\langle|\xi|^{p-2} \xi-|\zeta|^{p-2} \zeta, \xi-\zeta\right\rangle & \\
\times\left(|\xi|^{p}+|\zeta|^{p}\right)^{(2-p) / 2} & \text { for } 1<p<2
\end{array}\right.
$$

that

$$
\left\|u_{n}-u_{0}\right\|_{X} \longrightarrow 0 \quad \text { as } n \longrightarrow \infty \text {. }
$$

Then, we complete the proof.

\section{Existence of Solution}

In this section, the proof of Theorem 3 is mainly based on the following Mountain Pass lemma [17] (also see [18]).

Lemma 7 (Mountain Pass lemma). Let $X$ be a Banach space and $J \in C^{1}(X, \mathbb{R})$ satisfies $(P S)_{c}$ condition. Suppose that $J(0)=$ 0 and

$$
\begin{aligned}
& \left(I_{1}\right) \text { there are constant } \rho, \alpha>0 \text { such that } J_{\partial B_{\rho}} \geq \alpha, \\
& \left(I_{2}\right) \text { there is an } e \in X \backslash B_{\rho} \text { such that } J(e) \leq 0 .
\end{aligned}
$$

Then, J possesses a critical value $c \geq \alpha$. Moreover, $c$ can be characterized as

$$
c=\inf _{g \in \Gamma} \max _{u \in g[0,1]} J(u) \geq \alpha>0,
$$

where

$$
\Gamma=\{g \in C([0,1], X): g(0)=0, g(1)=e\} .
$$

In view of Lemma 6 , the functional $J(u)$ satisfies the $(\mathrm{PS})_{c}$ condition. It is obvious that $J(0)=0$; then, in order to apply Lemma 7, we need only to prove that $J(u)$ satisfies the geometric conditions of the Mountain Pass lemma.

Lemma 8. Assume $\left(A_{1}\right)-\left(A_{2}\right)$. Then,

$\left(I_{1}\right)$ there exist $\rho, \alpha>0$ such that $J(u) \geq \alpha>0$ with $\|u\|_{X}=$ $\rho$,

$\left(I_{2}\right)$ there is an $e \in X$ such that $\|e\|_{X}>\rho$ and $J(e) \leq 0$.

Proof. It follows from $\left(A_{2}\right),\left(A_{3}\right)$, and the embedding in Lemma 4 that

$$
\begin{aligned}
J(u) \geq & \frac{a}{p} \int_{\mathbb{R}^{N}} g(x)|\nabla u|^{p} d x+\frac{b}{2 p}\left(\int_{\mathbb{R}^{N}} g(x)|\nabla u|^{p} d x\right)^{2} \\
& +\frac{1}{p} \int_{\mathbb{R}^{N}} h(x)|u|^{p} d x-\frac{1}{\mu} \int_{\mathbb{R}^{N}}|u|^{q} d x \\
\geq & \frac{1}{p} \int_{\mathbb{R}^{N}}\left(g(x)|\nabla u|^{p}+h(x)|u|^{p}\right) d x-c\|u\|_{X}^{q} \\
= & \frac{1}{p}\|u\|_{X}^{p}-c\|u\|_{X}^{q} .
\end{aligned}
$$

Since $p<q$, there exists a sufficient small $\rho>0$ and $\alpha>0$ such that $J(u) \geq \alpha>0$ with $\|u\|_{X}=\rho$. On the other hand, for fixed $u_{0} \in X$ and $t>0$, it follows from $\left(A_{2}\right)$ that

$$
J\left(t u_{0}\right) \leq \frac{a t^{p}}{p} \int_{\mathbb{R}^{N}} g(x)|\nabla u|^{p} d x
$$

$$
\begin{aligned}
& +\frac{b t^{2 p}}{2 p}\left(\int_{\mathbb{R}^{N}} g(x)|\nabla u|^{p} d x\right)^{2}+\frac{t^{p}}{p} \int_{\mathbb{R}^{N}} h(x)|u|^{p} d x \\
& -t^{\mu} \int_{\mathbb{R}^{N}}\left|u_{0}\right|^{\mu} d x \longrightarrow-\infty \quad \text { as } t \longrightarrow+\infty .
\end{aligned}
$$


Then, there is a large $t_{0}>0$ such that $\left\|t_{0} u_{0}\right\|_{X}>\rho$ and $J\left(t_{0} u_{0}\right)<0$. One may choose $e=t_{0} u_{0}$; then, $\|e\|_{X}>\rho$ and $J(e)<0$. Thus, the proof of Lemma 8 is complete.

Proof of Theorem 3. It follows from Lemmas 7 and 8 that the functional $J(u)$ has a critical point $u$ such that $J(u)=c>0$; that is, problem (1) has at least one weak solution. On the other hand, $J(0)=0$; then, problem (1) has at least one nontrivial weak solution.

\section{Acknowledgment}

The author is very grateful to the anonymous reviewer for valuable comments and suggestions.

\section{References}

[1] G. Kirchhoff, Mechanik, Teubner, Leipzig, 1883.

[2] H. Bueno, G. Ercole, W. Ferreira, and A. Zumpano, "Existence and multiplicity of positive solutions for the $p$-laplacian with nonlocal coefficient," Journal of Mathematical Analysis and Applications, vol. 343, no. 1, pp. 151-158, 2008.

[3] F. J. S. A. Corrêa and G. M. Figueiredo, "On a $p$-kirchhoff equation via Krasnoselskii's genus," Applied Mathematics Letters, vol. 22, no. 6, pp. 819-822, 2009.

[4] F. J. S. A. Corrêa and G. M. Figueiredo, "On an elliptic equation of p-kirchhoff type via variational methods," Bulletin of the Australian Mathematical Society, vol. 74, no. 2, pp. 263-277, 2006.

[5] F. J. S. A. Corrêa and R. G. Nascimento, "On a nonlocal elliptic system of $p$-kirchhoff-type under Neumann boundary condition," Mathematical and Computer Modelling, vol. 49, no. 3-4, pp. 598-604, 2009.

[6] D. Liu and P. Zhao, "Multiple nontrivial solutions to a $p$ kirchhoff equation," Nonlinear Analysis. Theory, Methods \& Applications, vol. 75, no. 13, pp. 5032-5038, 2012.

[7] S. Heidarkhani, "Infinitely many solutions for systems of $n$ two-point kirchhoff-type boundary value problems," Annales Polonici Mathematici, vol. 107, no. 2, pp. 133-152, 2013.

[8] J. Jin and X. Wu, "Infinitely many radial solutions for Kirchhofftype problems in $\mathbb{R}^{N}$," Journal of Mathematical Analysis and Applications, vol. 369, no. 2, pp. 564-574, 2010.

[9] B. Ricceri, "On an elliptic kirchhoff-type problem depending on two parameters," Journal of Global Optimization, vol. 46, no. 4, pp. 543-549, 2010.

[10] F. Cammaroto, A. Chinnì, and B. Di Bella, "Some multiplicity results for quasilinear Neumann problems," Archiv der Mathematik, vol. 86, no. 2, pp. 154-162, 2006.

[11] S. Cingolani and G. Vannella, "On the multiplicity of positive solutions for $p$-laplace equations via Morse theory," Journal of Differential Equations, vol. 247, no. 11, pp. 3011-3027, 2009.

[12] M. Franca, "Radial ground states and singular ground states for a spatial-dependent $p$-laplace equation," Journal of Differential Equations, vol. 248, no. 11, pp. 2629-2656, 2010.

[13] A. Kristály and W. Marzantowicz, "Multiplicity of symmetrically distinct sequences of solutions for a quasilinear problem in $\mathbb{R}^{N}$," Nonlinear Differential Equations and Applications, vol. 15, no. 1-2, pp. 209-226, 2008.

[14] J. Su and Z.-Q. Wang, "Sobolev type embedding and quasilinear elliptic equations with radial potentials," Journal of Differential Equations, vol. 250, no. 1, pp. 223-242, 2011.
[15] S. B. Liu, "On ground states of superlinear $p$-Laplacian equations in $\mathbb{R}^{N}$," Journal of Mathematical Analysis and Applications, vol. 361, no. 1, pp. 48-58, 2010.

[16] J. I. Díaz, Nonlinear Partial Differential Equations and Free Boundaries, vol. 106 of Research Notes in Mathematics, Pitman Advanced Publishing, Boston, Mass, USA, 1985, Elliptic equations.

[17] A. Ambrosetti and P. H. Rabinowitz, "Dual variational methods in critical point theory and applications," Journal of Functional Analysis, vol. 14, pp. 349-381, 1973.

[18] M. Struwe, Variational Methods, Springer, Berlin, Germany, 3rd edition, 2000, Applications to nonlinear partial differential equations and Hamiltonian systems. 


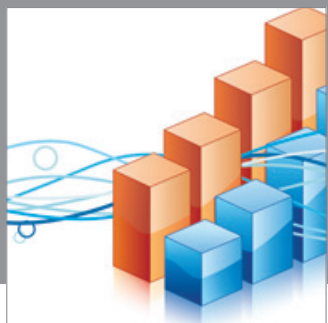

Advances in

Operations Research

mansans

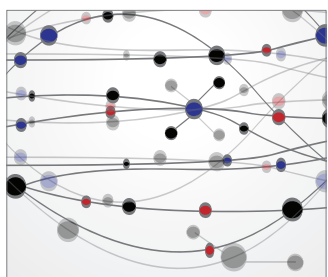

The Scientific World Journal
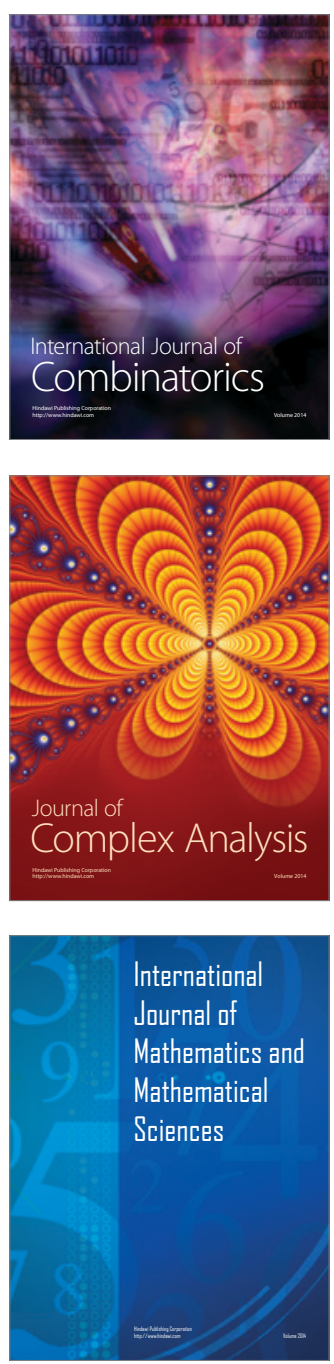
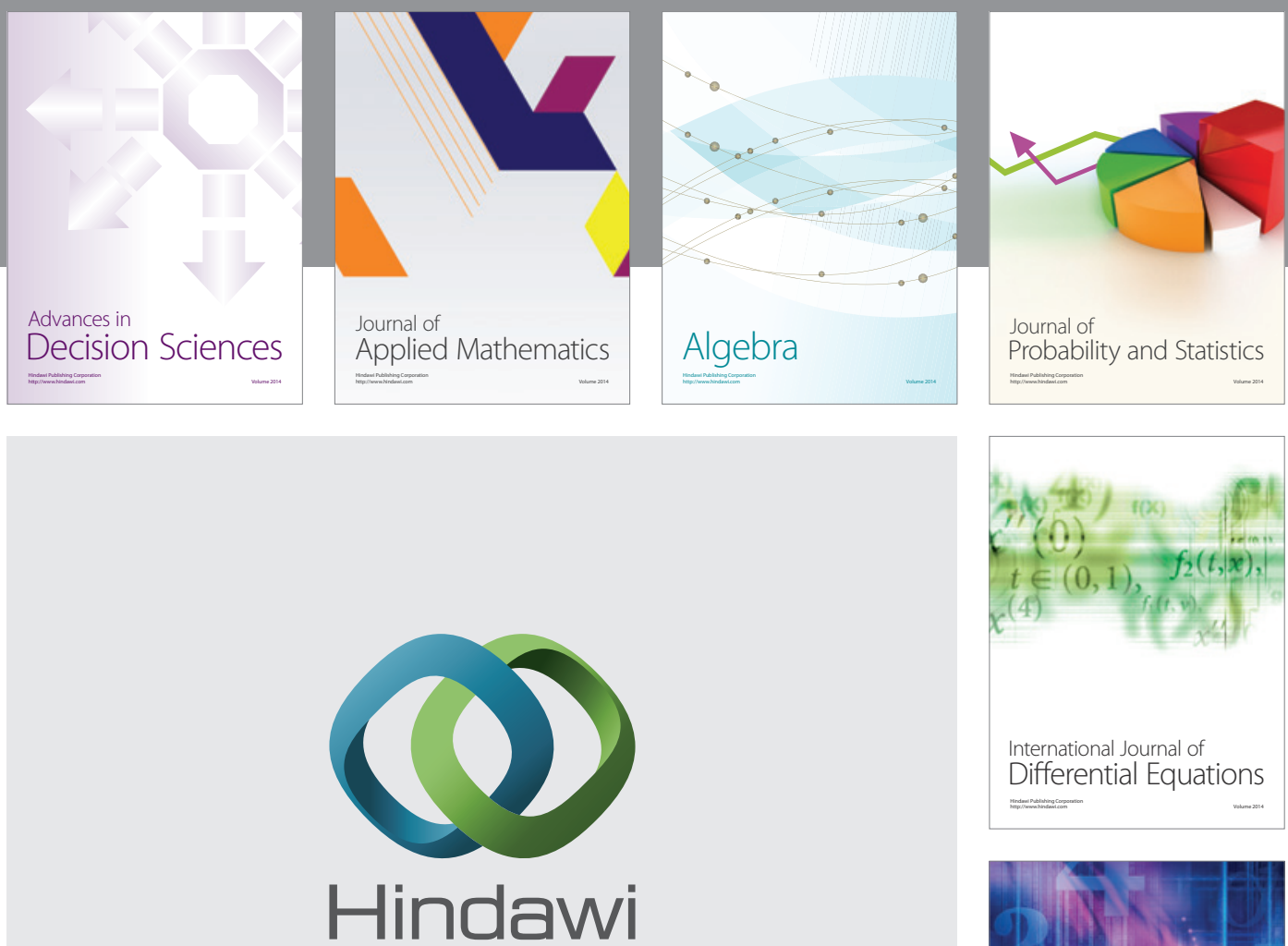

Submit your manuscripts at http://www.hindawi.com
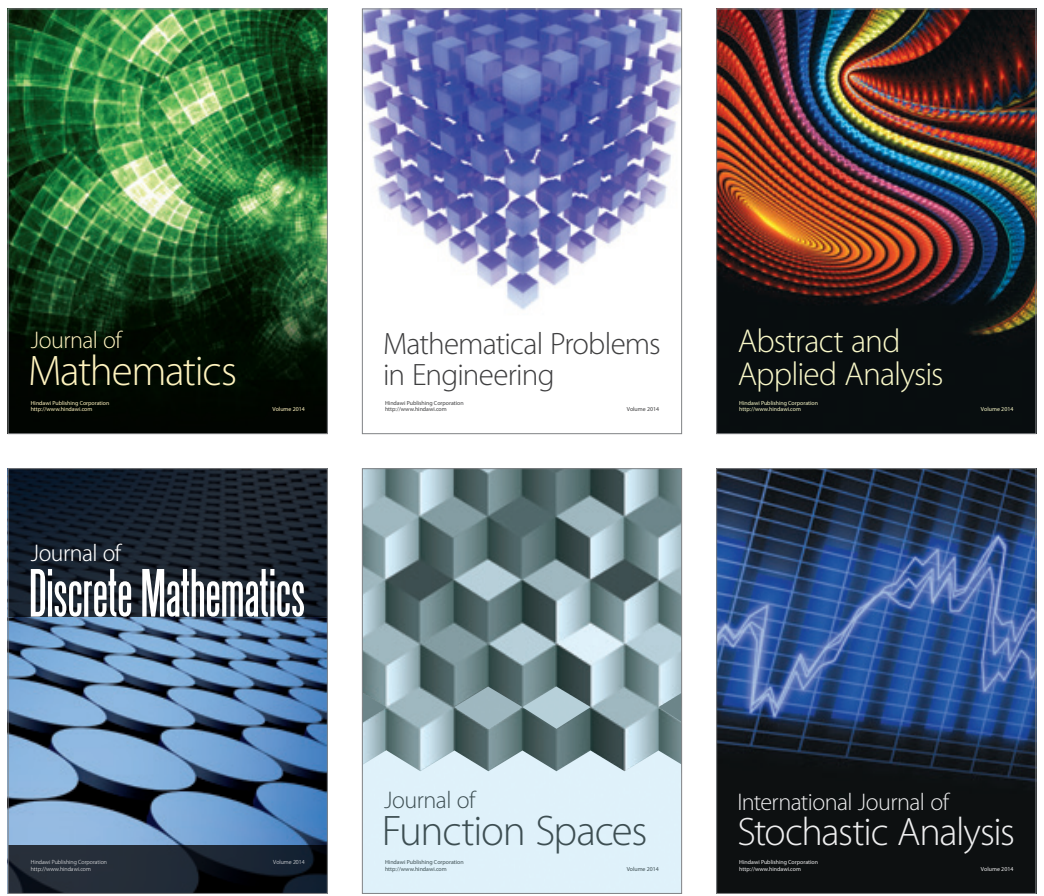

Journal of

Function Spaces

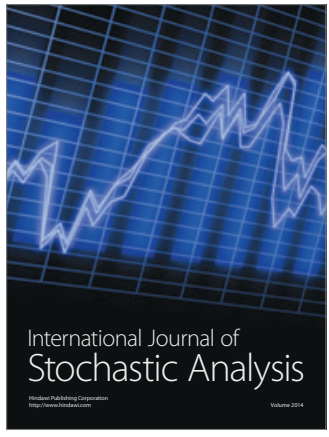

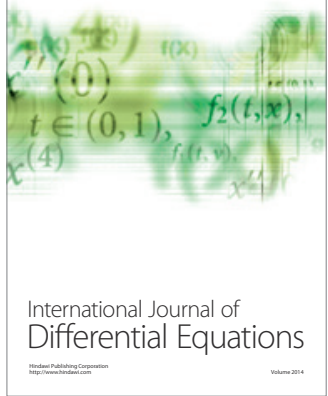
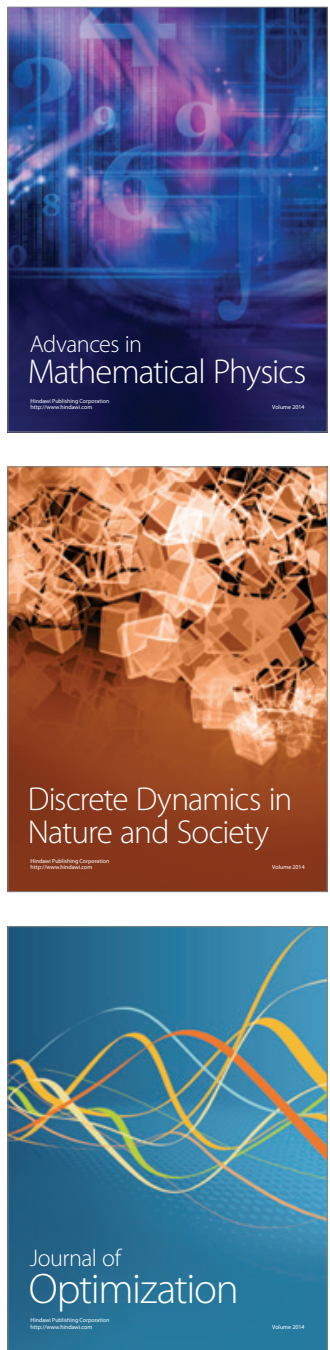